\title{
Robot Deployment with End-to-End Communication Constraints
}

\author{
Jerome Le Ny, Alejandro Ribeiro and George J. Pappas
}

\begin{abstract}
During deployment, mobile robots must form an ad-hoc wireless communication network guaranteeing reliable communications between agents and possibly with some fixed base stations. First, when only connectivity constraints need to be enforced, we discuss deployment algorithms whose execution does not require a model of the communication channels, but exploits possibly random and time-varying channel gain measurements between the robots to maintain a connected network. We then turn our attention to the more realistic situation where the communication network linking the robots must support certain rates between possibly distant terminals. For this problem, we propose deployment algorithms based on a projected-gradient scheme that provide end-to-end bandwidth guarantees, assuming a channel model with deterministic exponential path loss. In addition to setting the robot positions to optimize the deployment objective, these algorithms adjust the transmission powers at the wireless nodes and route communication packets through the network to support the desired flow rates.
\end{abstract}

\section{INTRODUCTION}

In the near future, teams of mobile robots will be used to perform autonomously a variety of intelligence, surveillance and reconnaissance missions, rescue missions, or transportation tasks. A prerequisite for realizing this vision however is to guarantee that reliable communication can be maintained with each vehicle in a deployed network. Moreover, the communication network should generally provide certain end-to-end quality of service guarantees. For example, an unmanned vehicle might have to transmit a video feed from its current position to a given base station, which requires maintaining a minimum communication rate between these two terminals.

In many scenarios, e.g. involving robot deployment underwater or in disaster or conflict areas, no external communication infrastructure is available. Therefore the robot team must establish the communication flows necessary to the accomplishment of the mission by forming an ad-hoc network. The problem of deploying a mobile robotic or sensor network under communication

\footnotetext{
This work was supported by the ONR-MURI award N00014-08-10696 and the ARL MAST-CTA award W911NF-08-2-0004.

The authors are with the Department of Electrical and Systems Engineering, University of Pennsylvania, Philadelphia, PA 19104, USA jeromel, aribeiro, pappasgeseas.upenn.edu.
}

constraints has been the object of much research, see e.g. [1]-[4]. Most of this work assumes very simple communication models, typically disc models with a fixed and known communication radius for each agent, and often a fixed or perhaps randomly varying topology for the communication network, in order to leverage geometric and graph theoretic techniques. Wireless channel models are notoriously difficult to characterize however [5], and in recent years, researchers have started using more realistic wireless communication models in robotic applications where ad-hoc networking problems and controlled mobility are coupled, see e.g. [6]-[12].

Our focus is on deployment problems, where a mobile robotic network attempts to reach a good steadystate spatial configuration, whose quality is measured by a scalar potential field to minimize, as with certain classical motion planners [13]-[15]. Deployment problems include source seeking [16], formation control [17], coverage control [18], and certain vehicle routing problems [19] for example. For concreteness, throughout the paper we frame the discussion in the context of a particular deployment problem, namely the adaptive coverage control problem discussed in [12].

Two types of communication constraints for the robotic network are discussed, for which we propose solutions that make increasingly strong assumptions about the wireless channel models. First, for the problem of maintaining connectivity between agents, we propose a simple adaptive algorithm that does not require any model of the communication channels, exploits measurements of received signal strength between the robots, and is robust to stochastic and time-varying fluctuations in the channel gain values. In contrast, algorithms relying on channel models are typically executed in an open-loop manner without ever actually testing the communication links between the robots. Next, for the more complex problem of maintaining communication rates between possibly distant pairs of agents, we resort to postulating a known path loss model for the wireless channels. It was pointed out in [6] that an exponential path-loss form for the model is useful for the joint optimization of wireless node locations and powers. With this assumption, we propose a projected-gradient heuristic that constrains the motion of the agents and 
sets transmission powers and routing parameters in order to satisfy the communication constraints during deployment. The projection step relies on a successive convex optimization approach, which can be implemented in a distributed way by using the techniques presented in e.g. [20], [21].

The rest of the paper is organized as follows. Section II discusses robot deployment problems and the particular adaptive coverage control algorithm used in the illustrative examples and simulations. In Section III we review some basic terminology and principles of wireless networking. Section IV discusses a penalizationbased adaptive algorithm that maintains connectivity between the robots in the network without relying on a model of the communication channels. Finally, Section $\mathrm{V}$ considers the problem of jointly optimizing the deployment configuration and the networking parameters to enforce end-to-end communication rate constraints.

\section{ADAPTIVE DEPLOYMENT}

Consider a mobile robotic network consisting of $n$ robots evolving in a workspace $\mathrm{W} \subset \mathbb{R}^{d}$, with positions denoted $\mathbf{x}=\left[\mathbf{x}_{1}, \ldots, \mathbf{x}_{n}\right] \in \mathrm{W}^{n}$. For simplicity, we assume throughout the paper that $\mathrm{W}$ is compact and convex. We denote $[n]=\{1, \ldots, n\}$. Some of the robot positions might be fixed, so that these "robots" can also in fact represent access points of a communication infrastructure, with which the mobile elements must maintain certain communication flows. We consider high-level motion planning problems where we neglect the dynamics of the robots, whose positions evolve in discrete time as

$$
\mathbf{x}_{i}^{k+1}=\mathbf{x}_{i}^{k}+\mathbf{u}_{i}^{k}, \quad i \in[n]
$$

where $\mathbf{u}_{i}^{k}$ is the control input for robot $i$ at period $k$.

The quality of the deployment of the robotic network is captured by a potential field $G(\mathbf{x})$ [15], whose minimum corresponds to a desired steady-state configuration $\mathrm{x}^{*}$ for the network. Hence the deployment problem for this potential field consists in designing control laws allowing the system to reach $\mathrm{x}^{*}$ starting from an initial configuration $\mathbf{x}^{0}=\left[\mathbf{x}_{1}^{0}, \ldots, \mathbf{x}_{n}^{0}\right]$. Most multirobot controllers take a simple gradient form

$$
\mathbf{u}^{k}\left(\mathbf{x}^{k}\right)=-\gamma^{k} \nabla G\left(\mathbf{x}^{k}\right)
$$

where $\gamma^{k}$ are pre-specified stepsizes, which in general only leads to a critical point of $G$. Truncated versions of (1) can be used to account for velocity constraints but are omitted for simplicity of exposition, as they do not change the convergence results. Our discussion follows this gradient descent approach, as global minimization of $G$ for many multi-robot deployment problems is computationally intractable.

For concreteness, we focus here on the coverage control problem introduced by Cortés et al. [18]. Let us assume that an event occurs in the workspace at each period at some random position $Z^{k}$. The probability distribution of the events is denoted $\mathbb{P}_{Z}$. Alternatively, $Z^{k}$ can denote the position of a single target with ergodic Markov dynamics and $\mathbb{P}_{Z}$ is its stationary distribution. The coverage control problem consists in finding a steady-state configuration for the robotic network that minimizes the objective

$$
G(\mathbf{x})=\mathbb{E}_{Z}\left[\min _{i \in[n]} c\left(\left\|Z-\mathbf{x}_{i}\right\|\right)\right],
$$

where $\mathbb{E}_{Z}$ is the expectation operator corresponding to $\mathbb{P}_{Z}$ and $c$ is an increasing, continuously differentiable function. This objective measures the average "service" performance for the events assuming that an event is serviced by the robot closest to its location, say robot $i$, at a cost $c\left(\left\|Z-\mathbf{x}_{i}\right\|\right)$.

Following the gradient controller (1) to minimize the coverage control objective (2) requires the knowledge of the distribution $\mathbb{P}_{Z}$, which for many scenarios of interest is difficult to estimate or to compute. Recently, the authors introduced stochastic gradient controllers for multi-robot deployment problems [12], which do not require this knowledge and in many cases lead to simple distributed algorithms. Consider an artificial potential such as (2) which takes the form of an expected value $G(\mathbf{x})=\mathbb{E}_{\xi}[g(\mathbf{x}, \xi)]$, where $\xi$ is a random variable with unknown distribution $\mathbb{P}_{\xi}$. Assume that we can observe i.i.d. realizations $\xi^{k}$ of the random variable $\xi$, or that $\left\{\xi^{k}\right\}_{k \geq 0}$ is an ergodic Markov chain with stationary distribution $\mathbb{P}_{\xi}$. Then, provided that the identity $\nabla_{\mathbf{x}} \mathbb{E}_{\xi}[g(\mathbf{x}, \xi)]=\mathbb{E}_{\xi}\left[\nabla_{\mathbf{x}} g(\mathbf{x}, \xi)\right]$ holds, the stochastic controller

$$
\mathbf{u}^{k}\left(\mathbf{x}^{k}\right)=-\gamma^{k} \nabla_{\mathbf{x}} g\left(\mathbf{x}^{k}, \xi^{k}\right)
$$

leads asymptotically almost surely to a configuration $\mathbf{x}$, which under broad conditions is a local minimum of $G$, if the stepsizes satisfy the standard conditions $\sum_{k=0}^{\infty} \gamma^{k}=\infty, \sum_{k=0}^{\infty}\left(\gamma^{k}\right)^{2}<\infty$. In other words, we recover the asymptotic behavior of the deterministic controller (1), but now the scheme does not require the knowledge of $\mathbb{P}_{\xi}$. In particular, the stochastic controller (3) for the coverage control problem (2) takes the simple form [12], [22]

$$
\mathbf{x}_{i}^{k+1}= \begin{cases}\mathbf{x}_{i}^{k}+\mathbf{u}_{i, 1}^{k}\left(Z^{k}, \mathbf{x}_{i}^{k}\right), & \text { if } i=i_{*}^{k} \\ \mathbf{x}_{i}^{k}, & \text { otherwise }\end{cases}
$$


where

$$
\mathbf{u}_{i, 1}^{k}\left(Z^{k}, \mathbf{x}_{i}^{k}\right)=\gamma^{k} c^{\prime}\left(\left\|Z^{k}-\mathbf{x}_{i}^{k}\right\|\right) \frac{Z^{k}-\mathbf{x}_{i}^{k}}{\left\|Z^{k}-\mathbf{x}_{i}^{k}\right\|}
$$

and $i_{*}^{k}$ is the index of the closest robot to the $k^{t h}$ target location

$$
i_{*}^{k} \in \arg \min _{i \in[n]}\left\{\left\|Z^{k}-\mathbf{x}_{i}^{k}\right\|\right\}
$$

with ties resolved arbitrarily. Determining $i_{*}^{k}$ at each period in the robotic network can be done with a finite number of message exchanges using a simple Floodmin algorithm for example [23]. At each period, only the agent closest to the event is required to detect its location in order to implement this scheme [22].

Note that the controllers (3) or (4) replace the requirement of a model of the uncertainty distribution by actual measurements in the workspace. We call such algorithms adaptive deployment algorithms, by analogy with the algorithms developed in adaptive control and signal processing [24].

\section{WIRELESS NETWORKING}

For the deployed robotic network to maintain communication, the gradient controllers (1) or (3) need to be further constrained. Indeed, wireless communication rates are related to inter-robot distances due to path loss and interferences. Each robot acts as a wireless terminal that wishes to deliver packets to other robots and base stations for different application level flows, where a flow is associated to a given destination. Packets for flow $\phi$ are generated at robot $i$ at rate $a_{i}^{\phi}$, with $a_{i}^{\phi} \leq 0$ if $i$ is the destination of flow $\phi$ and $\sum_{i=1}^{n} a_{i}^{\phi}=0$. Robots communicate using a set of frequency tones $f \in \mathcal{F}$. For every frequency $f \in \mathcal{F}$ and pair $(i, j) \in[n]$, let $h_{i j}^{f, k}\left(\mathbf{x}_{i}, \mathbf{x}_{j}\right)$ denote the communication channel power gain at period $k$ from robot $i$ at position $\mathbf{x}_{i}$ to robot $j$ at position $\mathbf{x}_{j}$. In wireless communications, $\left\{h_{i j}^{f, k}\right\}_{i j}$ is modeled as the realization of a random vector $H^{f, k}$. Models of wireless channel gains generally take the form

$$
\begin{aligned}
\left.h_{i j}^{f, k}\left(\mathbf{x}_{i}, \mathbf{x}_{j}\right)\right|_{d B} & =10 \log _{10} h_{i j}^{f, k}\left(\mathbf{x}_{i}, \mathbf{x}_{j}\right) \\
& =l^{f}\left(\mathbf{x}_{i}, \mathbf{x}_{j}\right)+Y_{i j}^{f, k}
\end{aligned}
$$

where $Y_{i j}^{f, k}$ is a zero-mean random variable modeling fading and shadowing effects [5]. The function $l^{f}\left(\mathbf{x}_{i}, \mathbf{x}_{j}\right)$ represents a deterministic path loss component between positions $\mathbf{x}_{i}$ and $\mathbf{x}_{j}$ in the frequency band $f$.

The simplest notion of communication network integrity consists in keeping the channel gains between robots above a certain threshold in steady state. Interferences can be neglected if we assume that distinct robot pairs communicate on different frequency bands for example. Much of the robotic literature relies on the classical disc model, where $Y_{i j}^{k}=0$ in (7), and $l\left(\mathbf{x}_{i}, \mathbf{x}_{j}\right)=\mathbf{1}\left\{\left\|\mathbf{x}_{i}-\mathbf{x}_{j}\right\| \leq R_{c}\right\}$ for a known communication radius $R_{c}$. By assuming $R_{c}$ to be perfectly known, one can develop open-loop algorithms that plan the motion of the robots without testing the wireless connectivity. However, such models lack realism and robustness to unavoidable modeling errors. In Section IV, we describe a simple algorithm to reach a steadystate configuration with sufficiently strong communication links. It does not require the knowledge of a channel model such as (7), relying instead on actual signal strength measurements to guide the robots.

Enforcing simple connectivity constraints is often not sufficient to carry out the tasks of a mobile robotic network. In general, the robots need to transmit back information in real-time to other robots or base stations (e.g. audio and video feeds), and for this purpose certain end-to-end rate constraints need to be enforced for particular flows. We assume that in addition to properly choosing their positions, the robots can adjust their wireless transmission powers in order to enforce these additional communication requirements and limit the effects of inter-robot interferences. We thus consider in Section $\mathrm{V}$ a more general but model-based wireless network optimization problem that constrains the deployment problem of the previous section and lets us set specific communication rates between terminals.

For this problem, we neglect the stochastic component of the channel gains, i.e., let $Y_{i j}^{f, k} \equiv 0$ in (7), assuming essentially that $l^{f}\left(\mathbf{x}_{i}, \mathbf{x}_{j}\right)$ can be estimated reliably. Let $p_{i j}^{f, k}$ denote the power used by robot $i$ to send packets to robot $j$ at period $k$ on tone $f$, and let $p^{f, k}=\left\{p_{i j}^{f, k}\right\}_{i j}$. The powers and robot positions at each period $k$ are constrained as follows in order to support the required communication rates

$$
\begin{aligned}
& c_{i j}^{f, k} \leq C_{i j}^{f}\left(\mathbf{x}^{k}, p^{f, k}\right), \\
& c_{i j}^{k} \leq \sum_{f \in \mathcal{F}} c_{i j}^{f, k}, \\
& p_{i, \max } \geq \sum_{j=1}^{n} \sum_{f \in \mathcal{F}} p_{i j}^{f, k} \\
& a_{i}^{\phi} \leq \sum_{j=1}^{n} r_{i j}^{\phi, k}-\sum_{j=1}^{n} r_{j i}^{\phi, k} \\
& \sum_{\phi} r_{i j}^{\phi, k} \leq c_{i j}^{k} .
\end{aligned}
$$

Here $p_{i, \max }$ is the maximum power that can be transmitted by the wireless terminal on robot $i \in[n]$, 
$C_{i j}^{f}\left(\mathbf{x}^{k}, p^{f, k}\right)$ is the achievable communication capacity on the link between robots $i$ and $j$ for frequency $f, c_{i j}^{k}$ is the communication rate actually used on this link, and $r_{i j}^{\phi, k}$ is the rate used for flow $\phi$. The flow-balance equation (11) ensures that the packets of flow $\phi$ are properly delivered to their destination. Note in particular that to maintain a certain flow rate between two given terminals, we can split the flow along several routes linking these terminals. Refer to [21] for a more detailed discussion of the constraints (8)-(12), and to Section V where in particular the achievable capacities appearing in (8) are discussed in more details.

\section{ENFORCING CONNECTIVITY}

In this section we consider the problem of approximately enforcing an average minimum channel strength between neighbors in the robotic network. We assume that the topology of the network is fixed, with robot $i \in[n]$ initially able to communicate with its set of neighbors denoted $\mathcal{N}(i) \subset[n]$. For concreteness, the network topology chosen in the simulations is the Delaunay triangulation [25] of the initial configuration of the robots, see Fig. 1. Interferences between robots are ignored in this section, for example by assuming that distinct robot pairs communicate on different frequency bands. We omit the indication of the frequency tone $f$ from the notation for the channel gains $h_{i j}^{k}\left(\mathbf{x}_{i}, \mathbf{x}_{j}\right)$.

In order to reach a configuration that takes into account the connectivity constraints, we consider the following modified cost function for the coverage control problem

$$
\begin{aligned}
G(\mathbf{x})= & \mathbb{E}_{Z}\left[\min _{i \in[n]} c\left(\left\|Z-\mathbf{x}_{i}\right\|\right)\right] \\
& +\kappa_{1} \sum_{i=1}^{n} \sum_{j \in \mathcal{N}(i)}\left(-l\left(\mathbf{x}_{i}, \mathbf{x}_{j}\right)+h_{1}\right),
\end{aligned}
$$

where $\kappa_{1}$ and $h_{1}$ are constants. The second term penalizes low channel gains. Note in particular from (7) that $-l\left(\mathbf{x}_{i}, \mathbf{x}_{j}\right) \rightarrow+\infty$ when the channel gain tends to zero. The stochastic gradient controller (3) takes the form

$$
\mathbf{x}_{i}^{k+1}= \begin{cases}\mathbf{x}_{i}^{k}+\mathbf{u}_{i, 1}^{k}+\mathbf{u}_{i, 2}^{k}, & \text { if } i=i_{*}^{k} \\ \mathbf{x}_{i}^{k}+\mathbf{u}_{i, 2}^{k}, & \text { otherwise, }\end{cases}
$$

where $\mathbf{u}_{i, 1}^{k}$ and $i_{k}^{*}$ are given by (5), (6), and

$$
\begin{aligned}
\mathbf{u}_{i, 2}^{k}\left(\mathbf{x}^{k}\right)= & -\gamma^{k} \sum_{j \in \mathcal{N}(i)} \frac{\partial l}{\partial \mathbf{x}_{i}}\left(\mathbf{x}_{i}, \mathbf{x}_{j}\right) \\
& -\gamma^{k} \sum_{j: i \in \mathcal{N}(j)} \frac{\partial l}{\partial \mathbf{x}_{i}}\left(\mathbf{x}_{j}, \mathbf{x}_{i}\right) .
\end{aligned}
$$

Here the notation $\partial / \partial \mathbf{x}_{i}$ denotes the vector of partial derivatives with respect to the components of $\mathbf{x}_{i}$. Typically $l\left(\mathbf{x}_{i}, \mathbf{x}_{j}\right)$ and the neighborhoods are symmetric, e.g. because they depend only on the distance $\left\|\mathbf{x}_{i}-\mathbf{x}_{j}\right\|$, and the two terms in (15) are equal.

In practice we do not necessarily know the function $l$ but we can reconstruct an estimate of its gradient (15) using noisy finite differences based on the channel gain measurements $h^{k}$. In particular, as noted in [12], the simultaneous perturbation stochastic approximation idea [26] is very useful to reduce the amount of coordination necessary in a multi-robot system to obtain such a gradient estimate. In this scheme, robot $i \in[n]$ generates at period $k$ independently of the other robots a $d$ dimensional random vector $\Delta_{i}^{k}$ with entries $\Delta_{i}^{m, k}=$ $\pm 1, m=1, \ldots, d$, each with equal probabilities $1 / 2$. A period is then divided into two subperiods. In the first subperiod, all robots move to the positions $\mathbf{x}_{i}^{k}-\epsilon^{k} \Delta_{i}^{k}$, $i \in[n]$, and measure the channel strengths $h_{i j}^{1, k}$ with their neighbors. In the second subperiod, they move to the positions $\mathbf{x}_{i}^{k}+\epsilon^{k} \Delta_{i}^{k}$ and measure again the channel strengths $h_{i j}^{2, k}$ with their neighbors. Now consider two connected robots $i, j$, and the following expressions computed by robot $i$

$g_{i j}^{m, k}=\left(\left.h_{i j}^{1, k}\right|_{d B}-\left.h_{i j}^{2, k}\right|_{d B}\right) /\left(2 \epsilon^{k} \Delta_{i}^{m, k}\right), m=1, \ldots, d$,

$\hat{g}_{i j}^{m, k}=\left(\left.h_{j i}^{1, k}\right|_{d B}-\left.h_{j i}^{2, k}\right|_{d B}\right) /\left(2 \epsilon^{k} \Delta_{i}^{m, k}\right), m=1, \ldots, d$.

By a Taylor expansion of (16), assuming $l$ is twice continuously differentiable, we have

$$
\begin{aligned}
g_{i j}^{m, k}= & \frac{\partial l}{\partial x_{i}^{m, k}}\left(\mathbf{x}_{i}^{k}, \mathbf{x}_{j}^{k}\right)+\frac{\partial l}{\partial x_{j}^{m, k}}\left(\mathbf{x}_{i}^{k}, \mathbf{x}_{j}^{k}\right) \frac{\Delta_{j}^{m, k}}{\Delta_{i}^{m, k}} \\
& +\left(Y_{i j}^{1, k}-Y_{i j}^{2, k}\right) /\left(2 \epsilon^{k} \Delta_{i}^{m, k}\right)+b_{i j}^{m, k}
\end{aligned}
$$

where the term $b_{i j}^{m, k}$ is a small second-order bias. Now note that all the random terms have zero mean, due to the properties of $Y_{i j}$ and the choice of the random vectors $\Delta_{i}^{k}$. In other words, up to the small bias term, $g_{i j}^{m, k}$ is an unbiased gradient estimate of $\frac{\partial l}{\partial x_{i}^{m, k}}\left(\mathbf{x}_{i}^{k}, \mathbf{x}_{j}^{k}\right)$, and similarly $\hat{g}_{i j}^{m, k}$ is an unbiased gradient estimate of $\frac{\partial l}{\partial x_{i}^{m, k}}\left(\mathbf{x}_{j}^{k}, \mathbf{x}_{i}^{k}\right)$. Hence with two simultaneous random motions of the robots, robot $i$ can construct an estimate of the terms appearing in (15) by communicating only with its neighbors, and updates its location by replacing the gradient expressions in (15) by

$$
\mathbf{u}_{i, 2}^{k}\left(\mathbf{x}^{k}\right)=-\gamma^{k}\left(\sum_{j \in \mathcal{N}(i)} g_{i j}^{k}+\sum_{j: i \in \mathcal{N}(j)} \hat{g}_{i j}^{k}\right) .
$$




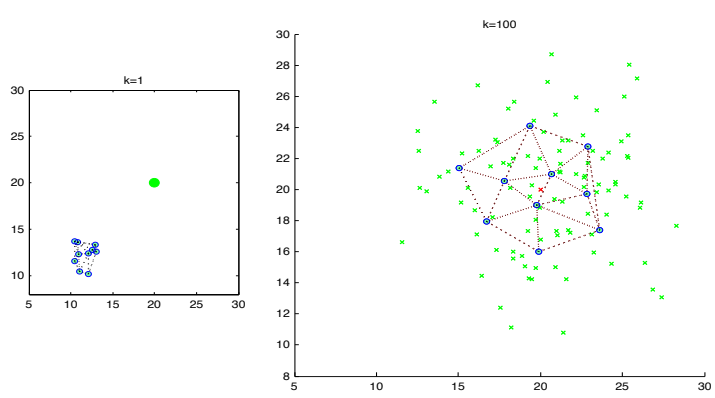

Fig. 1. Adaptive coverage control under stochastic wireless connectivity constraints. The topology of the network is fixed here to be the Delaunay triangulation for the initial configuration shown on the left figure. This figure also shows a green dot at the approximate mean of the distribution of the events. The figure on the right shows the configuration reached after 100 events occured. Here $\kappa_{1}=2, h_{1}=1$, and $\gamma^{k}=0.05+0.1 /(1+0.01 k)$. The asymptotically constant stepsizes ensure tracking of potential slow temporal variations in the target distribution but only provide convergence of the configurations to a neighborhood of a critical point of (13).
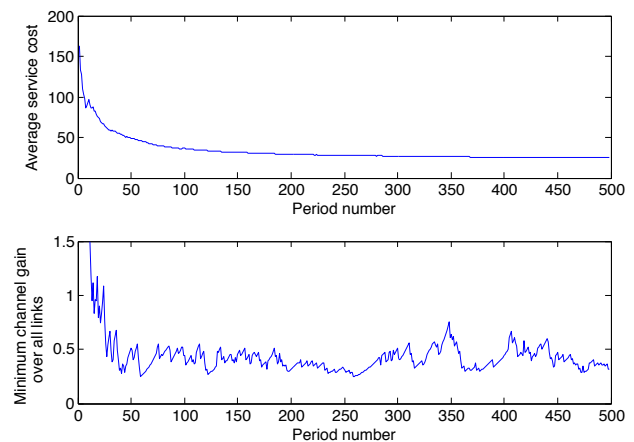

Fig. 2. Empirical average service time and and minimum channel gain value over all links for 500 periods in the adaptive coverage control scenario of Fig. 1.

Theorem 1: Assume that the robots follow Algorithm 1 , that the stepsizes $\gamma^{k}, \epsilon^{k}$ are positive, tend to 0 as $k \rightarrow \infty$, and satisfy

$$
\sum_{k=0}^{\infty} \gamma^{k}=\infty, \sum_{k=0}^{\infty}\left(\gamma^{k} / \epsilon^{k}\right)^{2}<\infty,
$$

and that $l$ is three times continuously differentiable. Then the sequence of configurations $\mathrm{x}^{k}$ converges almost surely to a critical point of the objective (13).

Proof: Follows from e.g. [27, chapter 7].

\section{Network Parameter Optimization}

We now turn to the problem of deploying robots while enforcing end-to-end rate constraints. Here our approach is different from the one used in the previous section and based on a projected gradient heuristic. We assume in this section a deterministic model of the channel gains, $\overline{\text { Algorithm } 1 \text { Connectivity-Constrained Adaptive Cover- }}$ age Control Algorithm

1: At period $k$, when a target appears at $Z^{k}$ :

2: Determine $i_{*}^{k}$ as in (6), e.g. via Floodmin

3: Each robot $1 \leq i \leq n$ moves to $\mathbf{x}_{i}^{k}-\epsilon^{k} \Delta_{i}^{k}$, measures the channel gain $h_{i j}^{1, k}$ with its neighbors and collects the values $h_{j i}^{1, k}$ from its neighbors.

4: Repeat 3 for $\mathbf{x}_{i}^{k}+\epsilon^{k} \Delta_{i}^{k}, h_{i j}^{2, k}, h_{j i}^{2, k}$.

5: Robot $i$ updates $\mathbf{x}_{i}^{k}$ according to (14), with $\mathbf{u}_{i, 2}^{k}$ given by (18).

6: Go to 1.

as in e.g. [6], [20]. Moreover, to simplify the joint power optimization and motion planning problem, we assume that $l^{f}$ takes the simple form

$$
l^{f}\left(\mathbf{x}_{i}, \mathbf{x}_{j}\right)=c_{1}^{\prime f}-c_{2}^{\prime f}\left\|\mathbf{x}_{i}-\mathbf{x}_{j}\right\|,
$$

which corresponds to an exponential path loss model, with $c_{1}^{\prime f}, c_{2}^{\prime f}$ known constants. Path loss models are in fact more often assumed to be of the form

$$
l\left(\mathbf{x}_{i}, \mathbf{x}_{j}\right)=c_{1}^{\prime \prime f}-10 c_{2}^{\prime \prime f} \log _{10}\left\|\mathbf{x}_{i}-\mathbf{x}_{j}\right\|,
$$

where $c_{1}^{\prime \prime f}, c_{2}^{\prime \prime f}$ are locally constant [5]. One possibility is to approximate locally a model such as (20) by the more conservative form (19), and to constrain the motions of the robots at each gradient step in the following algorithms to be limited to the trust region where the approximation is valid. In addition, the achievable capacity $C_{i j}^{f}(\mathbf{x}, p)$ of each link $(i, j)$ takes the form

$$
\begin{aligned}
C_{i j}^{f}\left(\mathbf{x}, p^{f}\right) & =K \ln \left(1+S I N R_{i j}^{f}\right) \\
S I N R_{i j}^{f} & =\frac{h_{i j}^{f}\left(\mathbf{x}_{i}, \mathbf{x}_{j}\right) p_{i j}^{f}}{\sigma_{j}^{f}+\sum_{(k, l) \in \mathcal{I}_{i j}^{f}} h_{k l}^{f}\left(\mathbf{x}_{k}, \mathbf{x}_{l}\right) p_{k l}^{f}},
\end{aligned}
$$

with $K$ a constant, $\sigma_{j}^{f}$ the receiver noise, $\mathcal{I}_{i j}^{f}$ the set of pairs $(k, l)$ interfering with packet transmissions between $i$ and $j$, and

$$
h_{i j}^{f}\left(\mathbf{x}_{i}, \mathbf{x}_{j}\right)=10^{l^{f}\left(\mathbf{x}_{i}, \mathbf{x}_{j}\right) / 10}, \forall i, j,
$$

see (7). Expression (21) assumes a capacity-achieving channel code.

The intermediate and asymptotic configurations of the robots must satisfy the constraints (8)-(12). We look for a final desired configuration which is solution to

$$
\min G(\mathbf{x})
$$

$$
\text { subject to }(8),(9),(10),(11),(12) \text {, }
$$

where $G(\mathbf{x})$ is the deployment objective. The variables are $\mathbf{x},\left\{p_{i j}^{f}\right\},\left\{r_{i j}^{\phi}\right\},\left\{c_{i j}^{f}\right\},\left\{c_{i j}\right\}$, and there are implicit positivity constraints on all but the position variables. 
The projected gradient algorithm used to minimize (23) operates as follows. Starting from some configuration $\mathrm{x}^{k}$ at period $k$, we first compute the unconstrained configuration

$$
\hat{\mathbf{x}}^{k+1}=\mathbf{x}^{k}-\gamma^{k} \nabla_{\mathbf{x}} G\left(\mathbf{x}^{k}\right)
$$

or its stochastic version (3). Then the iterate $\mathbf{x}^{k+1}$ is obtained by projection on the set of configurations satisfying the communication constraints, i.e., by solving the following auxiliary optimization problem

$$
\begin{aligned}
\min & \left\|\mathbf{x}-\hat{\mathbf{x}}^{k+1}\right\|^{2} \\
\text { subject to } & (8),(9),(10),(11),(12) .
\end{aligned}
$$

In general, this projection step is not easy to perform, due to the nonconvexity of the constraints (8). These constraints become convex under a high-SINR regime assumption which neglects the 1 in expression (21) [20]. However, this assumption usually leads to bad solutions for non-symmetric flows such as the ones often encountered in robotic networks. Indeed, consider a simple example with two nodes and node 2 only required to transmit to node 1 at a very small rate. Then the high-SINR assumption still forces the power $p_{21}$ to be relatively large for $S I N R_{21}$ to be much greater than one. As a result, we only make this assumption to obtain initial values for the decision variables in (23). We then follow a sequential convex optimization procedure to improve the solution for this projection step.

\section{A. Sequential Convex Optimization}

We first develop a convex approximation of the constraint (8). Let $\eta=1 / K$ and for simplicity of notation we drop the superscript $f$. Rewrite (8) as

$$
e^{\eta c_{i j}}-1 \leq \frac{e^{c_{1}-c_{2}\left\|\mathbf{x}_{i}-\mathbf{x}_{j}\right\|+\tilde{p}_{i j}}}{\sigma_{j}+\sum_{(k, l) \in \mathcal{I}_{i j}} e^{c_{1}-c_{2}\left\|\mathbf{x}_{k}-\mathbf{x}_{l}\right\|+\tilde{p}_{k l}}},
$$

with $\tilde{p}_{i j}=\ln p_{i j}$. The left hand side of this inequality is then approximated around some value of $c=\tilde{c}$, by using the Taylor expansion

$$
\ln \left(e^{\eta c_{i j}}-1\right) \approx \ln \left(e^{\eta \tilde{c}_{i j}}-1\right)+\left(c_{i j}-\tilde{c}_{i j}\right) \frac{\eta e^{\eta \tilde{c}_{i j}}}{e^{\eta \tilde{c}_{i j}}-1}
$$

so that

$$
e^{\eta c_{i j}}-1 \approx \alpha_{i j} e^{\beta_{i j} c_{i j}},
$$

with $\beta_{i j}=\frac{\eta e^{\eta \tilde{c}_{i j}}}{e^{\eta \tilde{c}_{i j}}-1}, \quad \alpha_{i j}=\left(e^{\eta \tilde{c}_{i j}}-1\right) e^{-\beta \tilde{c}_{i j}}$. Using this approximation (27), constraint (26) becomes

$$
\begin{aligned}
& \alpha_{i j} \sigma_{j} e^{\beta_{i j} c_{i j}}+\sum_{(k, l) \in \mathcal{I}_{i j}} \alpha_{i j} e^{c_{1}-c_{2}\left\|\mathbf{x}_{k}-\mathbf{x}_{l}\right\|+\tilde{p}_{k l}+\beta_{i j} c_{i j}} \\
& \leq e^{c_{1}-c_{2}\left\|\mathbf{x}_{i}-\mathbf{x}_{j}\right\|+\tilde{p}_{i j}} .
\end{aligned}
$$

We then introduce the variables

$$
\tilde{q}_{i j}=\tilde{p}_{i j}-c_{2}\left\|\mathbf{x}_{i}-\mathbf{x}_{j}\right\|, \quad \forall i, j,
$$

which can be interpreted as received powers [6]. We rewrite the constraint (28) and relax the identity (29) as

$$
\begin{aligned}
& \ln \left(\alpha_{i j} \sigma_{j} e^{-c_{1}+\beta_{i j} c_{i j}-\tilde{q}_{i j}}+\alpha_{i j} \sum_{k, l} e^{\tilde{q}_{k l}+\beta_{i j} c_{i j}-\tilde{q}_{i j}}\right) \leq 0 \\
& \tilde{q}_{k l}+c_{2}\left\|\mathbf{x}_{k}-\mathbf{x}_{l}\right\| \leq \tilde{p}_{k l}, \forall k, l \in[n] .
\end{aligned}
$$

These constraints are now convex, see [28, p.87]. The relaxation (30) is valid because if $\tilde{q}, \tilde{p}, \mathbf{x}$ is a solution of the problem (25) with the relaxed constraints (30), tightening these constraints is possible since the power variables are only involved in (10), a constraint that cannot be violated by reducing the transmission powers. Moreover, this tightening does not change the objective (25), which does not involve the power variables.

The sequential convex optimization approach attempts to reach the solution of (25) in several steps, as follows. Starting with an initial value $\tilde{c}$ for the capacity variables obtained say by convex programming under the highSINR assumption, we approximate the constraints (8) as explained above. The resulting convex problem is then solved to provide a new value for the capacity variables $\tilde{c}$ around which we approximate again (8) for the next iteration. The heuristic also fixes to zero the link capacities that are found to be zero during an iteration, since the approximation above is not valid for $\tilde{c}_{i j}=0$. We stop when the value between two successive iterations changes by less than a small threshold, or after a given number of iterations.

Note finally that in practice, we would need to solve the the projection step (25) in a distributed manner as well. For this purpose, a distributed algorithm can be derived based on the techniques presented in [21]. An example of final configuration obtained with this algorithm is shown on Fig. 3.

\section{CONCLUSION}

This paper has discussed robot deployment problems under wireless communication constraints. First, an adaptive algorithm is proposed for maintaining connectivity between robots by exploiting the possibility of measuring the channel gains during deployment. Second, we consider a joint deployment and wireless networking optimization problem, where rate constraints must be enforced between pairs of terminals. Under some assumptions on the wireless channel model, we described a projected gradient heuristic that provides good deployment configurations in practice. Future work will 


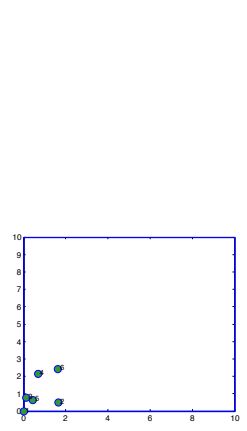

(a)

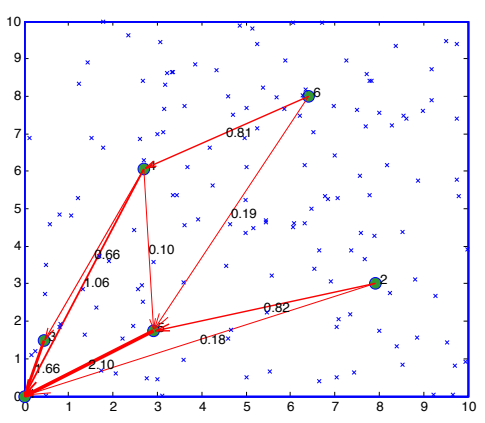

(b)
Fig. 3. Illustration of the adaptive coverage control algorithm with flow rate constraints. All the robots must transmit packets to the fixed base station at $(0,0)$ at a rate $a_{i}=1$ (there is a single flow). All robots transmit on the same frequency band, hence all pairs interfere with any given link. Fig. (a) shows the initial configuration, and Fig (b) shows the configuration obtained after the occurrence of 150 events. The width of the lines indicate the flow rates along that particular link, which is also indicated by numerical values.

refine this algorithm by incorporating channel sensing to handle a priori unknown channels.

\section{REFERENCES}

[1] J. Cortés, S. Martínez, and F. Bullo, "Spatially-distributed coverage optimization and control with limited-range interactions," ESAIM: Control, Optimisation and Calculus of Variations, vol. 11, no. 4, pp. 691-719, 2005.

[2] W. Li and C. G. Cassandras, "Distributed cooperative control of sensor networks," in Proceedings of the Conference on Decision and Control, Seville, Spain, December 2005, pp. 2542-2547.

[3] O. Burdakov, P. Doherty, K. Holmberg, J. Kvarnström, and P.-M. Olsson, "Positioning unmanned aerial vehicles as communication relays for surveillance tasks," in Proceedings of Robotics: Science and Systems, Seattle, USA, June 2009.

[4] M. Mesbahi and M. Egerstedt, Graph Theoretic Methods in Multiagent Networks. Princeton University Press, 2010.

[5] A. Goldsmith, Wireless Communications. Cambridge University Press, 2005.

[6] S. Firouzabadi and N. C. Martins, "Jointly optimal power allocation and constrained node placement in wireless networks of agents," University of Maryland, Tech. Rep., 2008.

[7] M. Lindhe, K. H. Johansson, and A. Bicchi, "An experimental study of exploiting multipath fading for robot communications," in Robotics: Science and Systems, Atlanta, GA, June 2007.

[8] E. M. Craparo, J. P. How, and E. Modiano, "Optimization of mobile backbone networks: Improved algorithms and approximation," in Proceedings of the American Control Conference, 2008, pp. 2016-2021.

[9] A. Ghaffarkhah and Y. Mostofi, "Communication-aware navigation functions for cooperative target tracking," in Proceedings of the American Control Conference, ST. Louis, MO, June 2009, pp. 1316-1322.

[10] M. M. Zavlanos, A. Ribeiro, and G. J. Pappas, "Mobility and routing control in networks of robots," in Proceedings of the 49th IEEE Conference on Decision and Control, Atlanta, GA, December 2009, pp. 7545-7550.

[11] J. Fink, A. Ribeiro, V. Kumar, and B. Sadler, "Optimal robust multihop routing for wireless networks of mobile micro autonomous systems," in Military Communications Conference, San Jose, CA, November 2010, pp. 1268-1273.
[12] J. Le Ny and G. J. Pappas, "Sensor-based robot deployment algorithms," in Proceedings of the IEEE Conference on Decision and Control, Atlanta, GA, December 2010.

[13] O. Khatib, "Real-time obstacle avoidance for manipulators and mobile robots," International Journal of Robotics Research, vol. 5, no. 1, pp. 90-98, 1986.

[14] E. Rimon and D. Koditschek, "Exact robot navigation using artificial potential functions," IEEE Transactions on Robotics and Automation, vol. 8, no. 5, pp. 501-518, October 1992.

[15] J. Reif and H. Wang, "Social potential fields: A distributed behavioral control for autonomous robots," Robotics and Autonomous Systems, vol. 27, pp. 171-194, 1999.

[16] P. Ögren, E. Fiorelli, and N. Leonard, "Cooperative control of mobile sensor networks: Adaptive gradient climbing in a distributed environment," IEEE Transactions on Automatic Control, vol. 49, no. 8, pp. 1292-1302, August 2004.

[17] R. Olfati-Saber, "Flocking for multi-agent dynamic systems: Algorithms and theory," IEEE Transactions on Automatic Control, vol. 51, no. 3, pp. 401-420, March 2006.

[18] J. Cortés, S. Martínez, T. Karatas, and F. Bullo, "Coverage control for mobile sensing networks," IEEE Transactions on Robotics and Automation, vol. 20, no. 2, pp. 243-255, April 2004.

[19] E. Frazzoli and F. Bullo, "Decentralized algorithms for vehicle routing in a stochastic time-varying environment," in Proceedings of the Conference on Decision and Control, 2004.

[20] M. Chiang, C. W. Tan, D. P. Palomar, D. O'Neill, and D. Julian, "Power control by geometric programming," IEEE Transaction on Wireless Communications, vol. 6, no. 7, pp. 2640-2651, July 2007.

[21] A. Ribeiro and G. Giannakis, "Separation principles in wireless networking," IEEE Transactions on Information Theory, vol. 56, no. 9, pp. 4488-4505, September 2010.

[22] J. Le Ny and G. J. Pappas, "Adaptive algorithms for coverage control and space partitioning in mobile robotic networks," University of Pennsylvania, Tech. Rep., October 2010, available at http://www.seas.upenn.edu/ jeromel/papers.html.

[23] N. A. Lynch, Distributed algorithms. Morgan Kaufmann, 1996.

[24] A. Benveniste, M. Metivier, and P. Priouret, Adaptive Algorithms and Stochastic Approximations. Springer, 1990.

[25] M. de Berg, O. Cheong, M. van Kreveld, and M. Overmars, Computational Geometry: Algorithms and Applications. SpringerVerlag, 2008.

[26] J. C. Spall, "Adaptive stochastic approximation by the simultaneous pertubation method," IEEE Transactions on Automatic Control, vol. 45, no. 10, pp. 1839-1853, October 2000.

[27] - Introduction to Stochastic Search and Optimization. Wiley, 2003.

[28] S. P. Boyd and L. Vandenberghe, Convex Optimization. Cambridge University Press, 2006. 